\title{
UN CAMPO SEMÁNTICO PARA LA MUERTE DESDE LA MIRADA ENFERMERA
}

\author{
A. M. Piera Orts
}

Lic. CC. de la Educación. Hospital General Universitario de Valencia

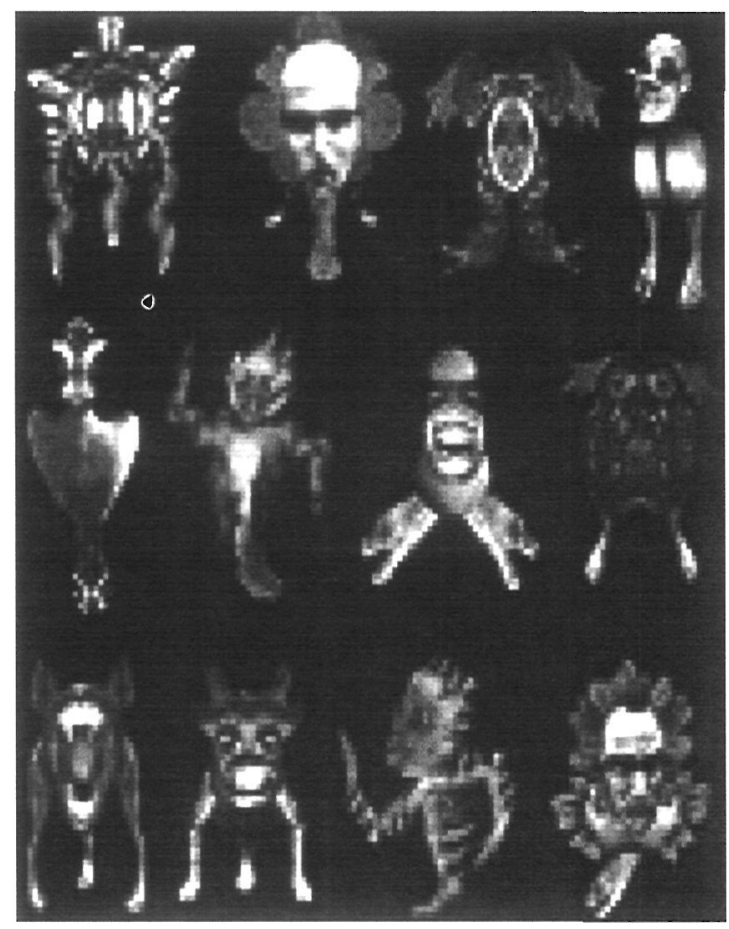

El título de este trabajo es propiamente un eufemismo, muestra de algunos más que aparecen en este texto. Ciertamente solemos aprender durante un período largo de años, el que dura nuestra vida académica como estudiantes, bajo diseños y estructuras de enseñanzas formales, pero también durante el resto de nuestras vidas, antes, durante y después de ese paréntesis académico, bajo estructuras no formales. Este es el resultado de un conocimiento formal "enganchado", "atrapado" en su inicio de modo no formal, experiencial, a través de la mirada hacia el otro. El formato para su presentación no se ajusta al convencional que explicita su metodología y sus fuentes como garantes de su validez, sino que toma la forma de carta reflexiva sobre un hecho

\section{RESUMEN}

La muerte, contemplada a diario en el hospital, llega a tomar una forma que, por común, se torna en ocasiones como un nuevo ámbito para un lenguaje lleno de eufemismos, generando una especie de campo semántico enfermero para pronunciar la muerte.

\section{SEMANTIC FIELDS FOR A DEATH FROM THE NURSING POINT OF VIEW}

\section{SUMMARY}

Death seen day after day in a hospital environment takes on a new shape, turning ordinary language into a speech full of euphemisms, creating a sort of special "nursing" semantic field which avoids the pronunciation of the word "death".

que, en cualquier momento, nos puede asaltar: ¿cómo se vive la muerte en un hospital?

No se trata de revisar la actitud ante la muerte y ante el morir, ante la incertidumbre sobre su hora, la discusión sobre el tema de la muerte solitaria que serian cuestiones abordables desde la gerontopsicología (DORSCH, 1994), como tampoco se trata de la necesidad de mirar hacia las propuestas de ayuda psicológicas para bien morir (WORCESTER, 1961). Tampoco se trata de establecer ningún contraste de la atención enfermera que reciben los pacientes terminales, como se dan en Unidades en las que se "convive" junto a la muerte como rezaba un reciente reportaje televisivo (TVE La 2 24.10.99) sino más bien de hacer 
visible uno de los mecanismos que, en cierto modo, serían explicables de un modo paralelo a cómo se hace desde la óptica de la expulsión de la muerte en la sociedad, con la consiguiente eliminación del ritual del duelo (GORER, 1965) y la pérdida de eficacia que comportaba éste, lo que encaja con el fenómeno de privatización del individuo, ese paso del llamado sujeto a individuo, como individuación y solitud.

El encuadre de esta cuestión se aleja de las concepciones de encarnizamiento terapéutico versus cuidados paliativos y se sitúa en al plano de la mismidad, término que aquí se re-inventa para tratar de representar la idea postmoderna de "un mí mismo pasivo, receptivo y cambiante que se representa exclusivamente la actividad de su propio pensamiento" (DELEUZE, 1996); un mí mismo que experimenta cambios en el tiempo, y que es como la expresión de un "yo pienso". Una idea algo descomprometida y aliviada, que basaría la explicación de lo circundante en mi "yo soy".

Esa mismidad sería la forma en que el individuo -el yo soy- se representase la vida, la enfermedad, el sufrimiento, el consuelo, el bienestar, etc. Unos códigos de orientación que constituyen, desde una óptica antropológica, referencias primigenias para la elección diaria de una dirección; son pues, construcciones categoriales que soportan una explicación sumergida que, si se sacan a la superficie, si se expresan, dan lugar a la reflexión sobre lo cotidiano. Una cotidianeidad que impregna de un modo cuasi imperceptible nuestro devenir diario y, por ende, nuestro ámbito profesional, combinándose los valores de la "conciencia" con los valores de la "eficacia" $y$, finalmente, se torna en comportamientos institucionalizados, como lo son los modos expresivos, las jergas profesionales, las técnicas y los malnombres.

Ciertamente se dan una serie de factores institucionales que desencadenan lo que podemos denominar efectos perversos de la propia institución. Uno de ellos es la propia organización hospitalaria que, al distribuir las responsabilidades por órganos y técnicas, viene a desintegrar, desde el punto de vista del paciente, la asistencia médica, efecto que se ve desde el enfoque organicista que señala al hospital como un "taller de cuerpos" tecnológico, y al tratamiento especialista como "mecánico del cuerpo" (COX y EWAN, 1990).
Otro de esos efectos es el hecho no explícito de considerar la muerte, en la medicina occidental, como un fracaso; mírese si no cómo se mide el éxito de una especialidad médica atendiendo a la relación del porcentaje de casos favorables frente a los que no responden a las aplicaciones tecnológicas y terapéuticas. Por lo tanto, no es de extrañar que algún tipo de profesional se vea a sí mismo como campeón de la vida, un campeón en situación de huida hacia adelante. Esa huida hacia adelante es la que en este trabajo se quiere recuperar y explicar desde la mismidad de quien lo relata, de ahí el uso de la primera persona.

Una vez sujetada la idea y recogidos los testimonios en torno a los cuales giraba este artículo, me fue necesario tomar un pequeño portafotos en forma hexagonal, que hace unos años llené con las fotografias del tronco y raíz de la familia de la que procedo. En él están todos los miembros de mi exigua familia, y cada uno de ellos muestra la imagen que tenían en el momento de mi vida en el que me sentí más vinculada a cada uno de ellos. Es por esa razón que alguno está por dos veces y otro incluso en la cama de un hospital, donde más seguro y cerca de la vida se sintió, mi padre. La sensación, tan repetida y conocida al tiempo, no tiene palabras que la describan más que como un pequeño bolo de aire seco que me oprime el esófago y me empuja los líquidos hacia los lagrimales hasta que estos, repletos, se expanden mejillas abajo. Es la vivificación física de la experiencia de la pérdida de un ser, mi mismidad. Esta pérdida se rememora una y otra vez a lo largo de las vivencias de semejante naturaleza, como una modalidad de preparación permanente a lo que inevitablemente tiene que acontecer.

Si los hospitales, representación del dolor y sufrimiento encerrado, causan una especie de estupor a los que no participan de su cotidianeidad, higienizada a través de unos elementos arquitectónicos y mobiliarios lavables y esterilizables, la muerte que tiene lugar en estos recintos cobra, parece, una dimensión un poco peculiar, de tal manera que llega a generar campos semánticos que, en ocasiones para el ajeno, suenan a una especie de boutade, pero que no tienen más función que la de separarse de esa realidad tan cruda, a través de una falsa construcción de identificadores lingüísticos por la vía de la traslación a otros territorios. Una 
especie de humoradas que reflejan la huida del hecho a través de una verbalización disfrazada.

Este disfraz lo buscamos ahora a partir de la reconstrucción de una historia que bien podría ser la de una sola enfermera, pero que pertenece a varios testimonios engarzados unos a otros y tomando la muerte como punto central.

Se los contaré así... Reparó en la muerte una noche de invierno... cuando tenía turno de noches... era muy joven todavía y jamás se había enfrentado a un hecho tan singular... por aquellas fechas la estructura de las habitaciones era de aquellas que tenían grandes dimensiones, en las que se ubicaban a ocho pacientes, con sus respectivos familiares... eran un poco lamentables, sobre todo para los pacientes que se hallaban más lejos de los ventanales porque, en ocasiones, el aire era irrespirable debido a la intensidad de algunos olores. Cada una de las camas disponía de una mesilla pequeñísima, una silla de hierro y un punto de luz situado junto al pulsador del timbre de llamada para enfermería. Había un paciente, el tercero de la derecha, que tenía el piloto encendido y que no cesaba de quejarse de un modo rítmico y algo quedo... jay madre, ay madre, ay madre...! pasaban las horas y no cambiaba el ritmo ni tampoco su empeño en mantener la luz encendida. Ella le dijo: -debería tratar de dormir un poco y desde luego callar un poquito además de apagar la luz, para que los otros pacientes puedan descansar-. -No, no puedo-, le contestó el hombre. -Pero, piense un poco, verdaderamente ¿siente alivio al decir tantísimas veces, ¡ay, ay, ay!?-, le preguntó ella. El hombre la miró y le dijo: -no, no siento alivio, pero necesito...-. -Bueno, al menos, permita que apaguemos el piloto para que no moleste a los demás pacientes y trate de dormir un poco-, le dijo ella. -No, si me duermo, ya no volveré a despertar -le dijo el hombre, en un tono absolutamente firme. Pero ella, pensando que los demás pacientes no resistían más y no creyendo la posibilidad de conocer la certeza del no despertar, le insistió: -no se preocupe, están aquí su hijo y su hermano, y yo estaré muy cerca, no tema, descanse un poco y verá como se le pasa ese malestar-. El hombre consintió finalmente en apagar la luz y se adormeció... ya no despertó jamás... su hijo salió corriendo dos horas después, sobre las cuatro de la madrugada; el hombre había muerto pasando de un sueño de reparación al sueño eterno... Veinte años después, ella siente la misma bola de aire opresivo cuando recuerda a aquel paciente cuyo nombre no llegó a anotarse para no olvidar, pero recuerda su temor a morir. Se dijo que nunca más permitiría que a su lado muriera nadie sin el consuelo de su mano próxima.

Ahora detecta varias posiciones frente a la muerte, sin entrar en las posturas de eticidad, que pertenecerían más bien al ámbito de la reflexión sobre la muerte cuando se describe desde una óptica de aprendizajes más o menos. Se refiere a una posición de vivificación directa cuando no hay más remedio que estar junto a ella y a otra posición que es la inmediata anterior o posterior al propio acontecimiento. ¿Cómo nos comemos un pastel unos minutos después de perder a un paciente?. Cuando la muerte se pasea con tanta proximidad parece un buen revulsivo burlarla con buenas dosis de apartamiento y ¿qué mejor que una pequeña burla?...

Al cambiar el turno en una Unidad de Enfermería, se leen desde luego los partes correspondientes a cada uno de los pacientes, pero por descontado que se comentan las cuestiones más inmediatas y urgentes. -¿Cómo está la sala?... ¡puf... hay dos pidiendo pista!... ¡no fastidies!, bah, no te preocupes que esta tarde no le toca turno a Laura y ella es quien tiene el gafe encima esta temporada... -.

-Bueno, te diré que realmente yo no puedo decir que haya visto morir a nadie en mi vida...-. - ¿Pero cómo dices eso trabajando en Cuidados Intensivos?...-; -pues sí... verdaderamente... están tan graves... siempre con tanto aparataje que, cuando mueren suele significar para mi, la retirada de sondas y tubos a alquien a quien no he llegado a ver con los ojos abiertos y muchas veces ni siquiera respirar por sí mismos.... de modo que no he visto la expresión de la muerte jamás...-. -Bien pero no deja de ser la muerte...-; -sí y no... porque no hay nada previo en el terreno de las emociones que yo advierta en los pacientes, ¿no ves que están en coma o muy sedados?... pues pasan de un tipo de sedación a otra...-

En el quirófano es diferente, digamos que hay también dos versiones de aproximación a un estado de no vida, donde la persona queda expresada por una serie de ritmos y constantes que se vigilan a través de un monitor. Son realidades humanas que nos sitúan en planos donde nuestra 
relación con el paciente queda alterada por algún efecto tecnológico. Mírese sino, cuál es la sensación que nos puede producir en un momento determinado, la mirada hacia un paciente que está fisiológicamente hibernado, al que, tras un cuidadoso control se le ha preparado para situarle en una parada cardio-circulatoria y un encefalograma plano antes de iniciar una perfusión cerebral, lo que posibilita el bombeo sanguíneo extracorpóreo o sin llegar a esta tecnificación, lo que nos supone mirar la simple dormición sin percepción de dolor, como efecto del proceso medicamentoso de la anestesia. En ambos casos, estamos ante la persona respondiente tan solo a través de mecanismos biológicos, pero sin posibilidad de conexión comunicativa hasta el despertar; un despertar que no todos relatan del mismo modo. Un despertar que en ocasiones se torna para el paciente en un grito demandando la reacción -Lucía, despierta, despierta o tendré que ponerte el respirador-; -oh que me está pasando no puedo contestar, los oigo, pero no noto siquiera si respiro... jtengo miedo....!. Unos mecanismos que llevan al paciente a una situación distante de nosotros mismos.

$\mathrm{Y}$ es, desde esa distancia, desde ese mí mismo pasivo, cuando decimos a nuestra compañera del turno siguiente, la 4.2 está a punto de cascar ... el 5.1 palmó anoche y menudo número.... el 3.2 está sepia... o incluso, la de la cama 8 está hecha una planta. Lo que no es más que un modo de huir hacia adelante, dejando atrás el duelo que se avecina, lo sobrepasamos y lo obviamos y si coincide con la hora de la merienda, tomaremos unos pasteles junto al café, en la salita de descanso, lugar protegido del dolor envolvente que se respira en el control de enfermería al que acude el familiar con la cara desencajada a pedir unas gasitas para limpiarle los labios al paciente.... Un familiar que a veces nos oye reír cuando estamos eligiendo entre el hojaldrado y la moka.

Habrá que tomar el pastel ¿por qué no?, pero tendríamos que reflexionar sobre la suficiencia o no de los pasos que se describen en ocasiones como un diagnóstico de enfermería: "impotencia relacionada con la inevitabilidad de la muerte, la falta de control sobre las funciones corporales..." ( VV.AA. 1994) y sobre los que se señalan unas actividades en ese tono que aporta la expresión de un verbo en infinitivo, como si la actividad cubriese realmente una necesidad detectada, como si no supiésemos de antemano que ese verbo en infinitivo, ese objetivo operativo, poco tiene de operativo y menos de acercamiento a un hecho antropológico tan evidente como es la atención personalizada a ese paciente, cuya vida se está agotando, y a ese familiar que se sienta junto a su cama a acompañar viviendo en ante duelo. Se habla de la experiencia vicaria de la muerte (MARQUES ANDRÉS, 1995), se habla también de la experiencia vivida de la pérdida de un otro importante (PILKINGTON, 1993) o de casos de muerte súbita y el escenario enfermero (EDWARDS, 1997), pero no se habla del impacto que causa en el entorno enfermero tanta muerte, como tampoco hemos recogido ningún testimonio de un familiar que, bajo situación de un sentimiento de pérdida, escuche en el pasillo a una enfermera decir: -éste está sepia-; es, cuando menos, como un doble golpe añadido al de pérdida, el de la infravalorización del ser, de la personeidad, en términos zubirianos, de ese sujeto que forma parte de la vida de quien escuchó la fatídica expresión. Quizás la reflexión podría empezar identificando la presencia de esa actuación basada en la mismidad, si es que es así y recuperar el concepto antropológico de hombre zubiriano en cuanto a sus ejes de personeidad, eso que se es, y como ese proceso en que la persona se va haciendo mientras vive (VILÁ PLADEVALL, 1998). No parece tener sentido vivir y hacerse personeidad, para acabar convertido a los ojos de la otreidad como una sepia, si es que nos cabe aquí el empleo de semejante comparación vulgar para una persona que sufre en un hospital y que pronto va a morir. Morir, dormir... para algunos.... tal vez aterrizar.

\section{BIBLIOGRAFÍA}

COX, K. y EWAN, C.E. (1990) La docencia en medicina. Ediciones DoymA, Barcelona.

DORSCH, F. (1996) Voz: Psicología de la muerte. En: Diccionario de Psicología. Editorial Herder. $1^{\mathrm{a}}$ reimpresión, Barcelona

EDWARDS, J. (1997) Sudden death and the theatre nurse. En: British Journal of Theatre Nursing. Mar. 6 (12): 11-14.

GORER. (1965) Voz: Psicología de la muerte. En: Diccionario de Psicología. Editorial Herder. $1^{\text {a }}$ reimpresión. Barcelona. 
MARQUES ANDRÉS, S. (1995) Morir. Tabúes, miedos, negaciones. En: Revista ROL de Enfermería. núm. 201: 17-21.

PILKINGTON, FB. (1993) The live experience of grieving the loss of an important other. En: Nursing Research Quaterly. 6 (3): 130-139.

VILÁ PLADEVALL, M. (1998) Las dimensiones de lo interhumano en la Antropología de X. Zubiri, consideradas en su apertura a la trascendencia. Ed. Facultad de Teología "San
Vicente Ferrer. Series Valentina XLII. Valencia.

VV.AA. (1994) Cuidados de enfermería a pacientes en hospitalización a domicilio. De. Generalitat Valenciana. Conselleria de Sanitat y Consum. Serie M, 19.

WORCESTER, (1961) Voz: Psicología de la muerte. En: Diccionario de Psicología. Editorial Herder. $1^{\mathrm{a}}$ reimpresión. Barcelona.

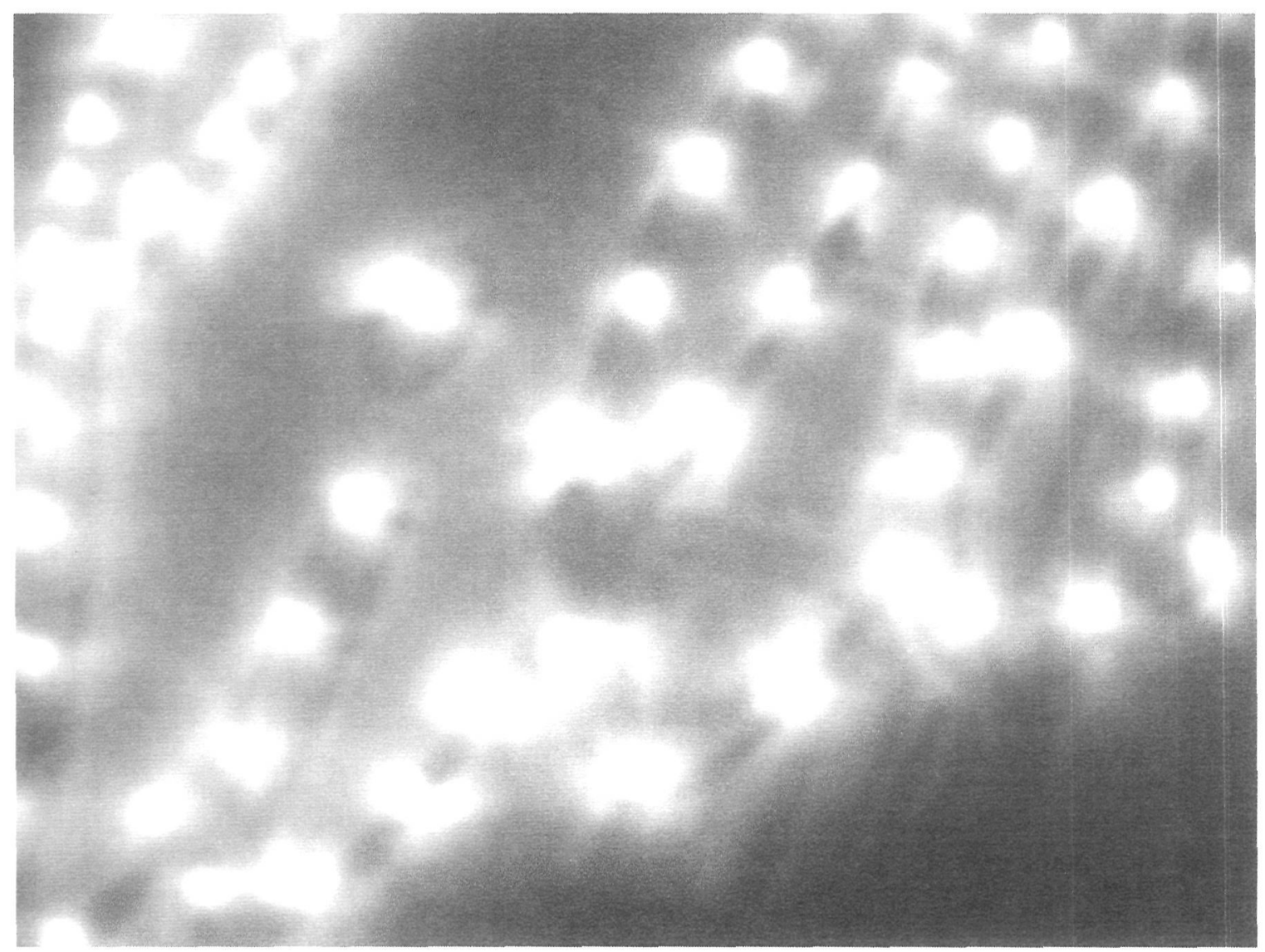

